International Journal of Pure and Applied Mathematics Volume 117 No. 1 2017, 69-80

ISSN: 1311-8080 (printed version); ISSN: 1314-3395 (on-line version)

url: http://www.ijpam.eu

doi: 10.12732/ijpam.v117i1.7

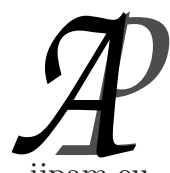

\title{
ISOMORPHISM ON ANTIFUZZY GRAPHS
}

\author{
R. Seethalakshmi ${ }^{1}$, R.B. Gnanajothi ${ }^{2}$ \\ ${ }^{1}$ Saiva Bhanu Kshatriya College \\ Aruppukottai, 626 101, Tamilnadu, INDIA \\ ${ }^{2}$ V.V. Vanniaperumal College \\ Virudhunagar, 626 001, Tamilnadu, INDIA
}

\begin{abstract}
The definition of complement of antifuzzy graphs is modified. The notion of isomorphism of antifuzzy graphs is introduced. Isomorphism between complement of join and union of two distinct antifuzzy graphs is discussed.
\end{abstract}

AMS Subject Classification: 05C72, 03E72

Key Words: antfuzzy graphs, complement of antifuzzy graphs, isomorphism on antifuzzy graphs

\section{Introduction}

The theory of fuzzy graphs was developed by Azriel Rosenfeld in 1975 [4]. Some operations of fuzzy graphs have been studied in [1] [2]. Sunitha and Vijayakumar [8] discussed about the complement of fuzzy graphs. Seethalakshmi and Gnanajothi [5] introduced the notion of antifuzzy graphs and discussed some of its properties. Nagoorgani and Malarvizhi [3] defined antipodal fuzzy graph and discussed its nature. Seethalakshmi and Gnanajothi [6], introduced antipodal antifuzzy graphs and discussed its properties. In this paper, isomorphism of antifuzzy graphs is introduced and it is discussed under various graph operations.

\begin{tabular}{lrc}
\hline Received: & $2017-06-06$ & (c) 2017 Academic Publications, Ltd. \\
Revised: & $2017-07-21$ & url: www.acadpubl.eu \\
Published: & November 28,2017 & \\
${ }^{\text {S Correspondence author }}$ &
\end{tabular}




\section{Preliminaries}

Definition 2.1. [5] Let $S_{1}$ and $S_{2}$ be two sets, $\sigma_{1}$ and $\sigma_{2}$ be fuzzy two sets of $S_{1}$ and $S_{2}$ respectively. That is $\sigma_{1}: S_{1} \rightarrow[0,1]$ and $\sigma_{2}: S_{2} \rightarrow[0,1]$. A function $\mu: S_{1} \times S_{2} \rightarrow[0,1]$ is called an anti-fuzzy relation on $\sigma_{1} \times \sigma_{2}$ if

$$
\mu(x, y) \geq \sigma(x) \vee \sigma(y), \forall x, y \in S_{1} \times S_{2},
$$

where $\vee$ denote the maximum.

Definition 2.2. [5] An anti-fuzzy graph $\mathcal{A}=(\sigma, \mu)$ is a pair of functions $\sigma: V \rightarrow[0,1]$ and $\mu: V \times V \rightarrow[0,1]$ with $\mu(u, v) \geq \sigma(u) \vee \sigma(v), \forall u, v \in V$, where $V$ is a finite non-empty set and $\vee$ denote maximum.

Definition 2.3. [5] The graph $\mathcal{A}^{*}=(V, E)$ is called the underlying crisp graph of the anti-fuzzy graph $\mathcal{A}$ where $V=\{u / \sigma(u)<1\}$ and $E=$ $\{(u, v) \in V \times V / \mu(u, v)<1\}$.

Definition 2.4. [5] An anti-fuzzy graph $\mathcal{A}=(\sigma, \mu)$ is said to be strong if $\mu(u, v)=\sigma(u) \vee \sigma(v), \forall(u, v) \in E$.

Definition 2.5. [5] An anti- fuzzy graph $\mathcal{A}=(\sigma, \mu)$ is said to be complete if the underlying graph $\mathcal{A}$ is complete and $\mu(u, v)=\sigma(u) \vee \sigma(v)$ for all $u, v \in V$.

Definition 2.6. [6] Let $\mathcal{A}=(\sigma, \mu)$ on $(V, E)$ be an antifuzzy graph. The $\mu$ - distance $\delta(u, v)$ is the smallest length of any $u-v$ path where $\mu$-length of a path $p: u_{0}, u_{1}, u_{2}, \cdots, u_{n}$ is $\delta(p)=\sum_{i=1}^{n} \mu\left(u_{i-1}, u_{i}\right)$

The eccentricity of a node $v$ is defined as $e(v)=\max \{\delta(u, v)\}$.

The diameter of $\mathcal{A}$ is $\operatorname{diam} \mathcal{A}=\vee\{e(v) / v \in V\}$.

The radius of $\mathcal{A}$ is $r(\mathcal{A})=\wedge\{e(v) / v \in V\}$.

A node whose eccentricity if minimum in a connected antifuzzy graph is called a central node.

A connected antifuzzy graph is called self centered if each node is a central node.

Definition 2.7. [6] Let $\mathcal{A}=(\sigma, \mu)$ on $(V, E)$ be an antifuzzy graph. Then the antipodal antifuzzy graph of $\mathcal{A}, A(\mathcal{A})=\left(\sigma_{A}, \mu_{A}\right)$ on $\left(V_{A}, E_{A}\right)$ is defined as follows:

The nodeset of $A(\mathcal{A})$ is the node set of $\mathcal{A}$.

Two nodes in $A(\mathcal{A})$ are made as neighbours if the $\mu$-distance between them is $\operatorname{diam} \mathcal{A}$. Therefore, $\sigma_{A}(u)=\sigma(u), \forall u \in V$.

If $\delta(u, v)=\operatorname{diam} \mathcal{A}$, then

$$
\left(\mu_{\mathcal{A}}\right)(u, v)=\left\{\begin{array}{l}
\mu(u, v) \text { if } u \text { and } v \text { are neighbours in } \mathcal{A} \\
\sigma(u) \vee \sigma(v) \text { if } u \text { and } v \text { are not neighbours in } \mathcal{A}
\end{array}\right.
$$


If $\delta(u, v) \neq \operatorname{diam} \mathcal{A}$ then $\mu_{A}(u, v)=1$

Definition 2.8. [5] Let $\mathcal{A}_{1}=\left(\sigma_{1}, \mu_{1}\right)$ on $\left(V_{1}, E_{1}\right)$ and $\mathcal{A}_{2}=\left(\sigma_{2}, \mu_{2}\right)$ on $\left(V_{2}, E_{2}\right)$ be two anti fuzzy graphs. Then the union of $\mathcal{A}_{1}$ and $\mathcal{A}_{2}$ is defined as $\mathcal{A}=\mathcal{A}_{1} \cup \mathcal{A}_{2}=\left(\sigma_{1} \cup \sigma_{2}, \mu_{1} \cup \mu_{2}\right)$ on $(V, E)$, where $V=V_{1} \cup V_{2}, E=E_{1} \cup E_{2}$.

$$
\left(\sigma_{1} \cup \sigma_{2}\right)(u)=\left\{\begin{array}{l}
\sigma_{1}(u) \quad \text { if } u \in V_{1}-V_{2} \\
\sigma_{2}(u) \text { if } u \in V_{2}-V_{1} \\
\min \left\{\sigma_{1}(u), \sigma_{2}(u)\right\} \text { if } u \in V_{1} \cap V_{2}
\end{array}\right.
$$

and

$$
\left(\mu_{1} \cup \mu_{2}\right)(u, v)=\left\{\begin{array}{l}
\mu_{1}(u, v) \text { if }(u, v) \in E_{1}-E_{2} \\
\mu_{2}(u, v) \text { if }(u, v) \in E_{2}-E_{1} \\
\min \left\{\mu_{1}(u, v), \mu_{2}(u, v)\right\} \text { if }(u, v) \in E_{1} \cap E_{2}
\end{array}\right.
$$

Definition 2.9. [5] Let $\mathcal{A}_{1}=\left(\sigma_{1}, \mu_{1}\right)$ on $\left(V_{1}, E_{1}\right)$ and $\mathcal{A}_{2}=\left(\sigma_{2}, \mu_{2}\right)$ on $\left(V_{2}, E_{2}\right)$ be two anti fuzzy graphs. Let $V=V_{1} \cup V_{2}$ and $E=E_{1} \cup E_{2} \cup E^{\prime}$ where $E^{\prime}$ is the set of edges joining of the nodes of $V_{1}$ and $V_{2}$. Assume that $V_{1} \cap V_{2} \neq \phi$. Then the join of $\mathcal{A}_{1}$ and $\mathcal{A}_{2}$ is $\mathcal{A}=(\sigma, \mu)=\left(\sigma_{1}+\sigma_{2}, \mu_{1}+\mu_{2}\right)$ on $(V, E)$ is defined as

$$
\begin{aligned}
\left(\sigma_{1}+\sigma_{2}\right)(u) & =\left(\sigma_{1} \cup \sigma_{2}\right)(u), \forall u \in V_{1} \cup V_{2} \text { and } \\
\left(\mu_{1}+\mu_{2}\right)(u, v) & =\left\{\begin{array}{l}
\left(\mu_{1} \cup \mu_{2}\right)(u, v) \text { if }(u, v) \in E_{1} \cup E_{2} \\
\max \left\{\sigma_{1}(u), \sigma_{2}(u)\right\} \text { if }(u, v) \in E^{\prime}
\end{array}\right.
\end{aligned}
$$

\section{Complement of Antifuzzy graph}

In our paper [5], Complement of antifuzzy graph was defined as:

Definition 3.1. Let $\mathcal{A}=(\sigma, \mu)$ on $(V, E)$ be an antifuzzy graph. Then the complement $\overline{\mathcal{A}}$ of $\mathcal{A}$ is defined as $\overline{\mathcal{A}}=(\bar{\sigma}, \bar{\mu})$, where $\bar{\sigma}=\sigma$ and $\bar{\mu}(u, v)=$ $1-\mu(u, v)+(\sigma(u) \vee \sigma(v)), \forall(u, v) \in E$.

Remark 3.2. In the above definition, $u, v \in V$ and $(u, v) \notin E$ are not taken care of. So, we modify the definition of complement as follows.

Definition 3.3. Let $\mathcal{A}=(\sigma, \mu)$ on $(V, E)$ be an antifuzzy graph. Then the complement $\overline{\mathcal{A}}$ of $\mathcal{A}$ is defined as $\overline{\mathcal{A}}=(\bar{\sigma}, \bar{\mu})$, where $\bar{\sigma}=\sigma$ and

$$
\bar{\mu}(u, v)=\left\{\begin{array}{l}
1-\mu(u, v)+(\sigma(u) \vee \sigma(v)) \text { for all } \quad(u, v) \in E \\
\sigma(u) \vee \sigma(v) \text { for }(u, v) \notin E
\end{array}\right.
$$


Theorem 3.4. If $\mathcal{A}$ is strong antifuzzy graph on a cycle, then $\mathcal{A} \cup \overline{\mathcal{A}}$ is complete antifuzzy graph.

Proof. Let $\mathcal{A}=(\sigma, \mu)$ be a strong antifuzzy graph on a cycle.

Then, $\mu(u, v)=\sigma(u) \vee \sigma(v), \forall(u, v) \in E$

Let $\overline{\mathcal{A}}=(\bar{\sigma}, \bar{\mu})$ be the complement of $\mathcal{A}$

Then, $\sigma=\bar{\sigma}$ and $\bar{\mu}(u, v)=1-\mu(u, v)+(\sigma(u) \vee \sigma(v))$

If, $(u, v) \in E$, then $\bar{\mu}(u, v)=1$ and

if $(u, v) \notin E$, then $\bar{\mu}(u, v)=\sigma(u) \vee \sigma(v)$ as $\mu(u, v)=1$.

Hence, the vertices which are not neighbours in $\mathcal{A}$ are neighbours in $\overline{\mathcal{A}}$

So, the underlying graph of $\mathcal{A} \cup \overline{\mathcal{A}}$ is complete and $\mathcal{A}$ is strong antifuzzy graph.

Since union of two disjoint strong antifuzzy graphs is again a strong antifuzzy graph, $\mathcal{A} \cup \overline{\mathcal{A}}$ is strong antifuzzy graph.

Hence, $\mathcal{A} \cup \overline{\mathcal{A}}$ is a complete antifuzzy graph.

Theorem 3.5. If $\mathcal{A}$ is any antifuzzy graph, the underlying graph of $\overline{\mathcal{A}}$ is complete if $\mu(u, v) \neq \sigma(u) \vee \sigma(v)$, for any $u, v \in V$.

Proof. Let $\mathcal{A}=(\sigma, \mu)$ be an antifuzzy graph on $(V, E)$ with $\mu(u, v) \neq$ $\sigma(u) \vee \sigma(v)$, for any $u, v \in V$.

Let $\overline{\mathcal{A}}=(\bar{\sigma}, \bar{\mu})$ be the complemet of $\mathcal{A}$

Case 1 Let $(u, v) \in E$.

Then $\mu(u, v)>\sigma(u) \vee \sigma(v)$. Therefore, $\sigma(u) \vee \sigma(v)-\mu(u, v)<0$.

Hence, $\bar{\mu}(u, v)>0$.

Case 2 Let $(u, v) \notin E$.

Then, $\mu(u, v)=1$. Therefore, $\bar{\mu}(u, v)=\sigma(u) \vee \sigma(v)>0$.

Hemce the underlying graph of $\overline{\mathcal{A}}$ is complete.

\section{Isomorphism of Antifuzzy Graphs}

Definition 4.1. Let $\mathcal{A}_{1}=\left(\sigma_{1}, \mu_{1}\right)$ on $\left(V_{1}, E_{1}\right)$ and $\mathcal{A}_{2}=\left(\sigma_{2}, \mu_{2}\right)$ on $\left(V_{2}, E_{2}\right)$ be two antifuzzy graphs.

1. A homomorphism $h: \mathcal{A}_{1} \rightarrow \mathcal{A}_{2}$ is a map $h: V_{1} \rightarrow V_{2}$ which satisfies $\sigma_{1}(u) \geq \sigma_{2}(h(u)), \forall u \in V$ and $\mu_{1}(u, v) \geq \mu_{2}(h(u), h(v)), \forall u, v \in V$.

2. An isomorphism $h: \mathcal{A}_{1} \rightarrow \mathcal{A}_{2}$ is a bijective map $h: V_{1} \rightarrow V_{2}$ which satisfies $\sigma_{1}(u) \geq \sigma_{2}(h(u)), \forall u \in V$ and $\mu_{1}(u, v) \geq \mu_{2}(h(u), h(v)), \forall u, v \in$ V.

Then $\mathcal{A}_{1}$ is said to be isomorphic to $\mathcal{A}_{2}$ 
3. A wead isomorphism $h: \mathcal{A}_{1} \rightarrow \mathcal{A}_{2}$ is a map $h: V_{1} \rightarrow V_{2}$ which is a bijective homomorphism that satisfies $\sigma_{1}(u)=\sigma_{2}(h(u), \forall u \in V$.

4. A co-wead isomorphism $h: \mathcal{A}_{1} \rightarrow \mathcal{A}_{2}$ is a map $h: V_{1} \rightarrow V_{2}$ which is a bijective homomorphism that satisfies $\mu_{1}(u, v)=\mu_{2}(h(u), h(v)), \forall u, v \in V$

Definition 4.2. $\mathcal{A}_{1}$ is said to be isometric with $\mathcal{A}_{2}$ if for each $v \in \mathcal{A}_{1}$, there is a bijection $\phi: V_{1} \rightarrow V_{2}$ such that $\delta_{1}(u, v)=\delta_{2}(\phi(u), \phi(v))$, for every $u \in V_{1}$.

Definition 4.3. An antifuzzy subgraph $\mathcal{B}=(\tau, \rho)$ is said to be a spanning antifuzzy subgraph of $\mathcal{A}=(\sigma, \mu)$ on $(V, E)$ if $\tau(u)=\sigma(u), \forall u \in V$.

In this case, two antifuzzy graphs have the same antifuzzy node set. They differ only in the arc weight.

Theorem 4.4. Let $\mathcal{A}_{1}=\left(\sigma_{1}, \mu_{1}\right)$ on $\left(V_{1}, E_{1}\right)$ and $\mathcal{A}_{2}=\left(\sigma_{2}, \mu_{2}\right)$ on $\left(V_{2}, E_{2}\right)$ be two antifuzzy graphs. If $\mathcal{A}_{1}$ is isomorphic to $\mathcal{A}_{2}$, then $\mathcal{A}_{1}$ is isometric with $\mathcal{A}_{2}$,

Proof. Since $\mathcal{A}_{1}$ is isomorphic to $\mathcal{A}_{2}$, there exist a bijection $h: V_{1} \rightarrow V_{2}$ such that $\sigma_{1}(u)=\sigma_{2}(h(u)), \forall u \in V_{1}$ and $\mu_{1}(u, v)=\mu_{2}(h(u), h(v)), \forall u, v \in V_{1}$

Also, for all $u \in V_{1}$,

$$
\begin{aligned}
\delta_{1}(u, v) & =\wedge\left\{\sum_{i=0}^{n} \mu_{1}\left(u_{i-1}, u_{i}\right)\right\}, \text { where } u_{0}=u, u_{n}=v \\
& =\wedge\left\{\sum_{i=0}^{n} \mu_{2}\left(h\left(u_{i-1}\right), h\left(u_{i}\right)\right)\right\} \\
& =\delta_{2}(h(u), h(v)), \quad \forall v \in V_{1}
\end{aligned}
$$

Hence, $\mathcal{A}_{1}$ is isometric with $\mathcal{A}_{2}$.

Observation. The above result is true even when $\mathcal{A}_{1}$ is coweak isomorphic to $\mathcal{A}_{2}$.

Theorem 4.5. Let $\mathcal{A}_{1}=\left(\sigma_{1}, \mu_{1}\right)$ on $\left(V_{1}, E_{1}\right)$ and $\mathcal{A}_{2}=\left(\sigma_{2}, \mu_{2}\right)$ on $\left(V_{2}, E_{2}\right)$ be two antifuzzy graphs. If $\mathcal{A}_{1}$ is isomorphic to $\mathcal{A}_{2}$, then $\overline{\mathcal{A}_{1}}$ is isomorphic with $\overline{\mathcal{A}_{2}}$,

Proof. Since $\mathcal{A}_{1}$ is isomorphic to $\mathcal{A}_{2}$, there exist a bijection $h: V_{1} \rightarrow V_{2}$ such that $\sigma_{1}(u)=\sigma_{2}(h(u)), \forall u \in V_{1}$ and $\mu_{1}(u, v)=\mu_{2}(h(u), h(v)), \forall u, v \in V_{1}$

Now, $\overline{\sigma_{1}}(u)=\overline{\sigma_{2}}(h(u))$, from definition.

$$
\text { Also, } \overline{\mu_{1}}(u, v)=1-\mu_{1}(u, v)+\left(\sigma_{1}(u) \vee \sigma_{1}(v)\right)
$$




$$
\begin{aligned}
& =1-\mu_{2}(h(u), h(v))+\left(\sigma _ { 2 } \left(h(u) \vee \sigma_{2}(h(v))\right.\right. \\
& =\overline{\mu_{2}}(h(u), h(v)), \quad \forall u, v \in V_{1} .
\end{aligned}
$$

Hence, $\overline{\mathcal{A}_{1}}$ is isomorphic with $\overline{\mathcal{A}_{2}}$. $\overline{\mathcal{A}_{2}}$

Remark. If $\mathcal{A}_{1}$ is isometric with $\mathcal{A}_{2}$, then $\overline{\mathcal{A}_{1}}$ need not be isometric with

\section{Example 4.6.}

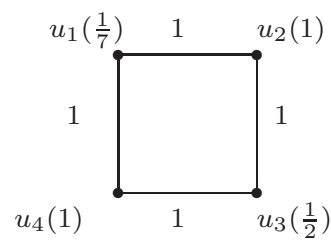

$\mathcal{A}_{1}$

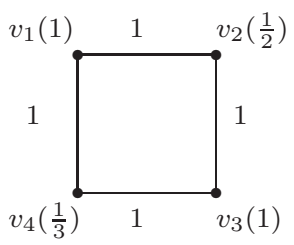

$\mathcal{A}_{2}$

Define $\phi: V_{1} \rightarrow V_{2}$ such that $\phi\left(u_{1}\right)=v_{1}, \phi\left(u_{2}\right)=v_{2}, \phi\left(u_{3}\right)=v_{3}$ and $\phi\left(u_{4}\right)=v_{4}$.

Then $\phi$ is one-one and onto.

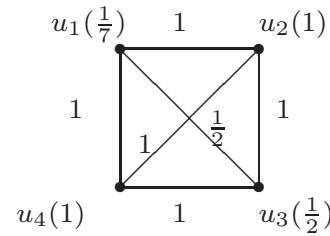

$\overline{\mathcal{A}_{1}}$

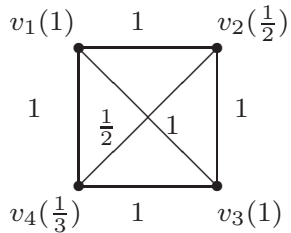

$\overline{\mathcal{A}_{2}}$

Here, $\overline{\mathcal{A}_{1}}$ is not isometric with $\overline{\mathcal{A}_{2}}$.

Theorem 4.7. Let $\mathcal{A}=(\sigma, \mu)$ on $(V, E)$ be a complete antifuzzy graph. Then $A(\mathcal{A})$ is a spanning subgraph of $\mathcal{A}$ and weight of each edge in $A(\mathcal{A})$ is $\vee\{\sigma(v) / v \in V\}$

Proof. Proof is analogous to theorem 4.5 in [3]

Theorem 4.8. Antipodal antifuzzy graphs of any two coweak isomorphic complete antifuzzy graphs are coweak isomorphic. 
Proof. Let $\mathcal{A}_{1}=\left(\sigma_{1}, \mu_{1}\right)$ on $\left(V_{1}, E_{1}\right)$ and $\mathcal{A}_{2}=\left(\sigma_{2}, \mu_{2}\right)$ on $\left(V_{2}, E_{2}\right)$ be two complete antifuzzy graphs. Let $\mathcal{A}_{1}$ is coweak isomorphic to $\mathcal{A}_{2}$.

Then there exists a bijection $h: \mathcal{A}_{1} \rightarrow \mathcal{A}_{2}$ satisfying

$$
\sigma_{1}\left(v_{1}\right) \geq \sigma_{2}\left(h\left(v_{1}\right)\right), \mu_{1}\left(v_{i}, v_{j}\right)=\mu_{2}\left(h\left(v_{i}\right), h\left(v_{j}\right)\right), \forall v_{i}, v_{j} \in V_{1}
$$

Let $V_{1}=\left\{v_{1}, v_{2}, \cdots, v_{n}\right\}$.

Arrange these vertices of $V_{1}$ such that $\sigma_{1}\left(v_{1}\right) \leq \sigma_{1}\left(v_{2}\right) \leq \cdots \sigma_{1}\left(v_{n}\right)$.

Since $\mathcal{A}_{1}$ and $\mathcal{A}_{2}$ are complete coweak isomorphic antifuzzy graphs, we have $\sigma_{1}\left(v_{i}\right)=\sigma_{2}\left(h\left(v_{i}\right)\right)$, for $i=1,2, \cdots, n-1$. and $\sigma_{1}\left(v_{n}\right) \geq \sigma_{2}\left(h\left(v_{n}\right)\right)$

Hence, $\mu_{1}\left(v_{i}, v_{j}\right)=\mu_{2}\left(h\left(v_{i}\right), h\left(v_{j}\right)\right), \forall v_{i}, v_{j} \in V_{1}$.

From theorem $4.7, A\left(\mathcal{A}_{i}\right)$ is a spanning antifuzzy graph of $\mathcal{A}_{i}$ with weights of each edge in $A\left(\mathcal{A}_{i}\right)$ as $\wedge\left\{\sigma_{i}(v) / v \in V_{i}\right\}_{i=1,2, \cdots, n}$.

Hence the same bijection $h$ is a coweak isomorphism between $A\left(\mathcal{A}_{1}\right)$ and $A\left(\mathcal{A}_{2}\right)$

Theorem 4.9. If $\mathcal{A}_{1}$ and $\mathcal{A}_{2}$ are connected antifuzzy graphs such that $\mathcal{A}_{1}$ is coweak isomorphic to $\mathcal{A}_{2}$, then $A\left(\mathcal{A}_{1}\right)$ is homomorphic to $A\left(\mathcal{A}_{2}\right)$

Proof. Let $\mathcal{A}_{1}=\left(\sigma_{1}, \mu_{1}\right)$ on $\left(V_{1}, E_{1}\right)$ and $\mathcal{A}_{2}=\left(\sigma_{2}, \mu_{2}\right)$ on $\left(V_{2}, E_{2}\right)$ be connected antifuzzy graphs.

Suppose $\mathcal{A}_{1}$ is coweak isomorphic to $\mathcal{A}_{2}$ Then there exists a bijection $h$ : $\mathcal{A}_{1} \rightarrow \mathcal{A}_{2}$ satisfying $\sigma_{1}\left(v_{i}\right) \leq \sigma_{2}\left(h\left(v_{i}\right)\right)$, and $\mu_{1}\left(v_{i}, v_{j}\right)=\mu_{2}\left(h\left(v_{i}\right), h\left(v_{j}\right)\right)$, for $v_{i}, v_{j} \in V_{1}$

Also, $\mu$ distance will be preserved.

Let daim $\mathcal{A}_{1}=\operatorname{diam} \mathcal{A}_{2}=k$ (say)

If $u, v \in V_{1}$, are at a distance $k$ in $\mathcal{A}_{1}$, then they are made neighbours in $A\left(\mathcal{A}_{1}\right)$

So, $h(u), h(v)$ in $\mathcal{A}_{2}$ are also at a distance $k$ in $\mathcal{A}_{2}$ and $h(u), h(v)$ are made as neighbours in $A\left(\mathcal{A}_{2}\right.$, where $\sigma$ attains its maximum at a unique vertex.

If $u$ and $v$ are neighbours in $\mathcal{A}_{1}$, then

$\mu_{\mathcal{A}_{1}}(u, v)=\mu_{1}(u, v)=\mu_{2}(h(u), h(v))=\mu_{\mathcal{A}_{2}}(h(u), h(v))$

If $u$ and $v$ are not neighbours in $\mathcal{A}_{1}$, then

$\mu_{\mathcal{A}_{1}}(u, v)=\sigma_{1}(u) \vee \sigma_{1}(v) \leq \sigma_{2}(h(u)) \vee \sigma_{2}(h(v))=\mu_{\mathcal{A}_{2}}(h(u), h(v))$

Hence, $A\left(\mathcal{A}_{1}\right)$ is homomorphic to $A\left(\mathcal{A}_{2}\right)$.

Theorem 4.10. Let $\mathcal{A}=(\sigma, \mu)$ on $(V . E)$ be an antifuzzy graph on a cycle $C_{n}$ with $\mu(u, v)=c, \forall(u, v) \in E$, where $0<c<1$. Then

1. $\mathcal{A}$ is self centered antifuzzy graph.

2. $A(\mathcal{A})$ is strong antifuzzy graph. 
3. $A(\mathcal{A})$ is a spanning subgraph of $\overline{\mathcal{A}}$.

Proof. Let $\mathcal{A}=(\sigma, \mu)$ be an antifuzzy graph on a cycle $C_{n}$.

1) Let $V=\left\{v_{1}, v_{2}, \cdots, v_{n}\right\}$ be the vertices of the cycle $C_{n}$.

Now, $\delta\left(v_{i}, v_{i-1}\right)=\delta\left(v_{i}, v_{i+1}\right)=c, \forall i=1,2, \cdots, n$.

$\delta\left(v_{i}, v_{i-2}\right)=\delta\left(v_{i}, v_{i+2}\right)=2 c, \forall i=1,2, \cdots, n$.

$\delta\left(v_{i}, v_{i-3}\right)=\delta\left(v_{i}, v_{i+3}\right)=3 c, \forall i=1,2, \cdots, n$.

$\delta\left(v_{i}, v_{i-\frac{n}{2}}\right)=\delta\left(v_{i}, v_{i+\frac{n}{2}}\right)=\frac{n}{2} c$, if $n$ is even.

$\delta\left(v_{i}, v_{i-\left\lfloor\frac{n}{2}\right\rfloor}\right)=\delta\left(v_{i}, v_{i+\left\lceil\frac{n}{2}\right\rceil}\right)=\left[\frac{n}{2}\right] c$, if $n$ is odd, where $\left\lfloor\frac{n}{2}\right\rfloor$ is flooring of $\frac{n}{2}$ and $\left\lceil\frac{n}{2}\right\rceil$ is ceiling of $\frac{n}{2}$.

Hence,

$$
e\left(v_{i}\right)=\max \left\{\delta\left(v_{i}, v_{j}\right)\right\}=\left[\frac{n}{2}\right] c, \forall i=1,2, \cdots, n .
$$

Therefore, radius of $\mathcal{A}=\left[\frac{n}{2}\right] c=\operatorname{diam} \mathcal{A}$.

So, each node is a central node.

Hence, $\mathcal{A}$ is self centered antifuzzy graph.

2) Since $\operatorname{diam} \mathcal{A}=\left[\frac{n}{2}\right] c$, the nodes $v_{i}$ and $v_{i-\frac{n}{2}}$ and $v_{i}$ and $v_{i+\frac{n}{2}}$ are neighbours in $A(\mathcal{A})$ which are not neighbours in $\mathcal{A}$, if $n$ is even.

The nodes $v_{i}$ and $v_{i-\left\lfloor\frac{n}{2}\right\rfloor}$ and $v_{i}$ and $v_{i+\left\lceil\frac{n}{2}\right\rceil}$ are neighbours in $A(\mathcal{A})$ which are not neighbours in $\mathcal{A}$, if $n$ is odd.

From the definition of antipodal antifuzzy graph,

$\mu\left(v_{i}, v_{i-\frac{n}{2}}\right)=\sigma\left(v_{i}\right) \vee \sigma\left(v_{i-\frac{n}{2}}\right)$ and $\mu\left(v_{i}, v_{i+\frac{n}{2}}\right)=\sigma\left(v_{i}\right) \vee \sigma\left(v_{i+\frac{n}{2}}\right)$, if $n$ if even. and if odd.

$\mu\left(v_{i}, v_{i-\left\lfloor\frac{n}{2}\right\rfloor}\right)=\sigma\left(v_{i}\right) \vee \sigma\left(v_{i-\left\lfloor\frac{n}{2}\right\rfloor}\right)$ and $\mu\left(v_{i}, v_{i+\left\lceil\frac{n}{2}\right\rceil}\right)=\sigma\left(v_{i}\right) \vee \sigma\left(v_{i+\left\lceil\frac{n}{2}\right\rceil}\right)$, if $n$

Hence, $A(\mathcal{A})$ is strong antifuzzy graph.

3) From the definition of complement and antipodal antifuzzy graph, $\sigma(v)=\bar{\sigma}(v)=\sigma_{A}(v), \forall v \in V$.

Hence, $A(\mathcal{A})$ is spanning subgraph of $\overline{\mathcal{A}}$.

\section{Self Complementary Antifuzzy Graph}

Definition 5.1. An Antifuzzy graph is self complementary if $\mathcal{A} \cong \overline{\mathcal{A}}$

Theorem 5.2. Let $\mathcal{A}=(\sigma, \mu)$ be a self complementary antifuzzy graph on $(V, E)$. Then

$$
\sum_{u \neq v} \mu(u, v)=\frac{1}{2}\left(\left(\begin{array}{l}
\gamma \\
2
\end{array}\right)+\sum_{u \neq v} \sigma(u) \vee \sigma(v)\right)
$$


, where $\gamma=|V|$

Proof. As $\mathcal{A}$ is a self complementary antifuzzy graph, there exists an isomorphism $f: V \rightarrow V$ such that $\bar{\sigma}(f(u))=\sigma(u), \forall u \in V$ and $\bar{\mu}(f(u), f(v))=$ $\mu(u, v), \forall u, v \in V$

As $\overline{\mathcal{A}}$ is complement of $\mathcal{A}, \sigma=\bar{\sigma}$ and

$\bar{\mu}(f(u), f(v))=1-\mu(f(u), f(v))+(\bar{\sigma}(u) \vee \bar{\sigma}(v)), \forall u, v \in V$

Therefore, $\mu(u, v)=1-\mu(f(u), f(v))+(\sigma(u) \vee \sigma(v)), \forall u, v \in V$

$\Rightarrow \mu(u, v)+\mu(f(u), f(v))=1+(\sigma(u) \vee \sigma(v))$ Hence,

$$
\sum_{u \neq v} \mu(u, v)+\sum_{u \neq v} \mu(f(u), f(v))=\sum_{u \neq v} 1+\sum_{u \neq v}(\sigma(u) \vee \sigma(v))
$$

Therefore

$$
2 \sum_{u \neq v} \mu(u, v)=\left(\begin{array}{l}
\gamma \\
2
\end{array}\right)+\sum_{u \neq v}(\sigma(u) \vee \sigma(v)), \text { where } \gamma=|V|
$$

Hence,

$$
\sum_{u \neq v} \mu(u, v)=\frac{1}{2}\left(\left(\begin{array}{l}
\gamma \\
2
\end{array}\right)+\sum_{u \neq v}(\sigma(u) \vee \sigma(v))\right)
$$

Remark 5.3. The condition of the above theorem is not sufficient.

\section{Example 5.4.}

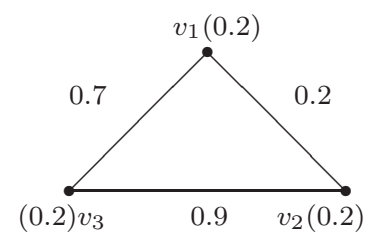

$\mathcal{A}$

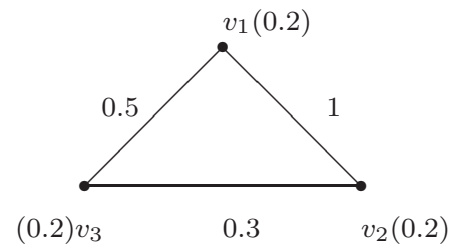

$\overline{\mathcal{A}}$

Here,

$$
\sum_{u \neq v} \mu(u, v)=0.2+0.9+0.7=1.8
$$

Also,

$$
\frac{1}{2}\left(\left(\begin{array}{l}
\gamma \\
2
\end{array}\right)+\sum_{u \neq v} \sigma(u) \vee \sigma(v)\right)=\frac{1}{2}(3+0.2+0.2+0.2)=1.8 .
$$

But $\mathcal{A}$ is not isomorphic to $\overline{\mathcal{A}}$. 
Theorem 5.5. Let $\mathcal{A}=(\sigma, \mu)$ be an antifuzzy graph on $(V, E)$. If $\mu(u, v)=$ $\frac{1}{2}(1+(\sigma(u) \vee \sigma(v))), \forall u, v \in V$, then $\mathcal{A}$ is self complmentary.

Proof. Let $\mu(u, v)=\frac{1}{2}(1+(\sigma(u) \vee \sigma(v))), \forall u, v \in V$.

Then $\mathcal{A}$ is isomorphic to $\overline{\mathcal{A}}$ under the identity map on $V$.

Hence, $\bar{\mu}(u, v)=1-\mu(u, v)+(\sigma(u) \vee \sigma(v))=\mu(u, v)$.

$\Rightarrow \mathcal{A} \cong \overline{\mathcal{A}}$

Illustration of Theorem 5.5.

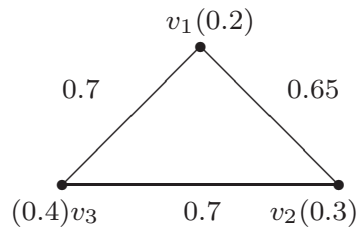

$\mathcal{A}$

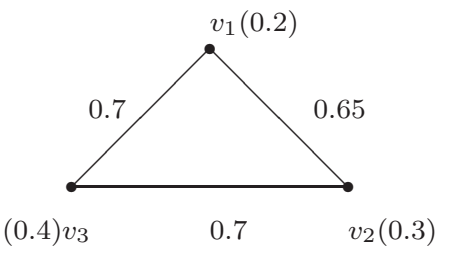

$\overline{\mathcal{A}}$

Remark 5.6. The condition in theorem 5.5 is not necessary.

\section{Example 5.7.}
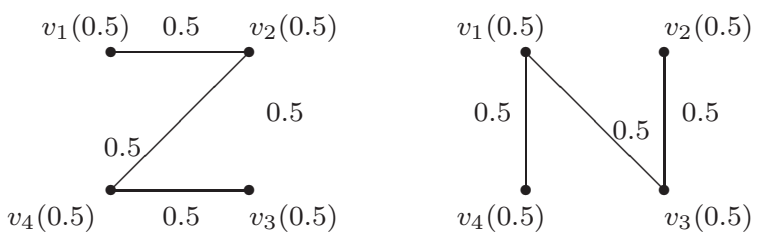

$\mathcal{A}$

Here, $\mathcal{A} \cong \overline{\mathcal{A}}$ under the isomorphism $f: V \rightarrow V$ given by $f\left(v_{1}\right)=v_{2} ; f\left(v_{2}\right)=v_{3} ; f\left(v_{3}\right)=v_{4} ; f\left(v_{4}\right)=v_{1}$

Also, $\mu\left(v_{1}, v_{2}\right)=0.5$. But, $\frac{1}{2}\left(1+\left(\sigma\left(v_{1} \vee \sigma\left(v_{2}\right)\right)\right)=\frac{1}{2}(1+0.5)=\frac{1.5}{2}=0.75\right.$.

So, $\mu\left(v_{1}, v_{2}\right) \neq \frac{1}{2}\left(1+\left(\sigma\left(v_{1} \vee \sigma\left(v_{2}\right)\right)\right)\right.$.

Theorem 5.8. Let $\mathcal{A}_{1}=\left(\sigma_{1}, \mu_{1}\right)$ on $\left(V_{1}, E_{1}\right)$ and $\mathcal{A}_{2}=\left(\sigma_{2}, \mu_{2}\right)$ on $\left(V_{2}, E_{2}\right)$ be two disjoint antifuzzy graphs. Then

1. $\overline{G_{1}+G_{2}} \cong \overline{G_{1}} \cup \overline{G_{2}}$

2. $\overline{G_{1} \cup G_{2}} \cong \overline{G_{1}}+\overline{G_{2}}$

Proof. We shall prove that the identity map is the required isomorphism.

Let $I: V_{1} \cup V_{2} \rightarrow V_{1} \cup V_{2}$ be the identity map. 
1) We have to prove that $\left(\overline{\sigma_{1}+\sigma_{2}}\right)(u)=\left(\overline{\sigma_{1}} \cup \overline{\sigma_{2}}\right)(u)$ and $\left(\overline{\mu_{1}+\mu_{2}}\right)(u, v)=\left(\overline{\mu_{1}} \cup \overline{\mu_{2}}\right)(u, v)$

Now,

$$
\begin{aligned}
\left(\overline{\sigma_{1}+\sigma_{2}}\right)(u) & =\left(\sigma_{1}+\sigma_{2}\right)(u), \text { from definition of complement } \\
& = \begin{cases}\sigma_{1}(u) & \text { if } u \in V_{1}-V_{2} \\
\sigma_{2}(u) & \text { if } u \in V_{2}-V_{1}\end{cases} \\
& = \begin{cases}\overline{\sigma_{1}}(u) & \text { if } u \in V_{1}-V_{2} \\
\overline{\sigma_{2}}(u) & \text { if } u \in V_{2}-V_{1}\end{cases} \\
& =\left(\overline{\sigma_{1}} \cup \overline{\sigma_{2}}\right)(u)
\end{aligned}
$$

Also,

$$
\begin{aligned}
& \left(\overline{\mu_{1}+\mu_{2}}\right)(u, v)=1-\left(\mu_{1}+\mu_{2}\right)(u, v)+\left(\left(\sigma_{1}+\sigma_{2}\right)(u) \vee\left(\sigma_{1}+\sigma_{2}\right)(v)\right) \\
& =\left\{\begin{array}{r}
1-\left(\mu_{1} \cup \mu_{2}\right)(u, v)+\left(\left(\sigma_{1} \cup \sigma_{2}\right)(u) \vee\left(\sigma_{1} \cup \sigma_{2}\right)(v)\right), \\
\text { if }(u, v) \in E_{1}-E_{2} \\
1-\left(\sigma_{1}(u) \vee \sigma_{2}(v)\right)+\left(\left(\sigma_{1} \cup \sigma_{2}\right)(u) \vee\left(\sigma_{1} \cup \sigma_{2}\right)(v)\right), \\
\text { if }(u, v) \in E_{1}^{\prime}
\end{array}\right. \\
& =\left\{\begin{aligned}
& 1-\mu_{1}(u, v)+\left(\sigma_{1}(u) \vee \sigma_{1}(v)\right), \\
& \text { if } u \in V_{1}-V_{2} \text { and }(u, v) \in E_{1}-E_{2} \\
& 1-\mu_{2}(u, v)+\left(\sigma_{2}(u) \vee \sigma_{2}(v)\right), \\
& \text { if } u \in V_{2}-V_{1} \text { and }(u, v) \in E_{2}-E_{1} \\
& 1-\left(\sigma_{1}(u) \vee\right.\left.\sigma_{2}(v)\right)+\left(\sigma_{1}(u) \vee \sigma_{2}(v)\right), \\
& \text { if } u \in V_{1}, v \in V_{2} \text { and }(u, v) \notin E
\end{aligned}\right. \\
& =\left\{\begin{array}{l}
\overline{\mu_{1}}\left(u, v \quad \text { if }(u, v) \in E_{1}-E_{2}\right. \\
\overline{\mu_{2}}(u, v) \text { if }(u, v) \in E_{2}-E_{1} \\
1 \quad \text { if }(u, v) \in E^{\prime}
\end{array}\right. \\
& =\left(\overline{\mu_{1}} \cup \overline{\mu_{2}}\right)(u, v)
\end{aligned}
$$

2) We have to prove that $\left(\overline{\sigma_{1} \cup \sigma_{2}}\right)(u)=\left(\overline{\sigma_{1}}+\overline{\sigma_{2}}\right)(u)$ and $\left(\overline{\mu_{1} \cup \mu_{2}}\right)(u, v)=\left(\overline{\mu_{1}}+\overline{\mu_{2}}\right)(u, v)$

Now,

$$
\begin{aligned}
\left(\overline{\sigma_{1} \cup \sigma_{2}}\right)(u) & =\left(\sigma_{1} \cup \sigma_{2}\right)(u) \\
& = \begin{cases}\sigma_{1}(u) & \text { if } u \in V_{1}-V_{2} \\
\sigma_{2}(u) & \text { if } u \in V_{2}-V_{1}\end{cases} \\
& =\left\{\begin{array}{lll}
\overline{\sigma_{1}}(u) & \text { if } u \in V_{1}-V_{2} \\
\overline{\sigma_{2}}(u) & \text { if } u \in V_{2}-V_{1}
\end{array}\right.
\end{aligned}
$$




$$
=\left(\overline{\sigma_{1}}+\overline{\sigma_{2}}\right)(u)
$$

We have, if $(u . v) \notin E$, then

$$
\left(\mu_{1} \cup \mu_{2}\right)(u, v)=1,\left(\sigma_{1} \cup \sigma_{2}\right)(u)=\sigma_{1}(u) \text { and }\left(\sigma_{1} \cup \sigma_{2}\right)(v)=\sigma_{2}(v) .
$$

Also,

$$
\begin{aligned}
\left(\overline{\mu_{1} \cup \mu_{2}}\right)(u, v)= & 1-\left(\mu_{1} \cup \mu_{2}\right)(u, v)+\left(\left(\sigma_{1} \cup \sigma_{2}\right)(u) \vee\left(\sigma_{1} \cup \sigma_{2}\right)(v)\right) \\
= & \left\{\begin{array}{r}
1-\mu_{1}(u, v)+\left(\sigma_{1}(u) \vee \sigma_{1}(v)\right), \\
\text { if } u \in V_{1}-V_{2} \text { and }(u, v) \in E_{1}-E_{2} \\
1-\mu_{2}(u, v)+\left(\sigma_{2}(u) \vee \sigma_{2}(v)\right), \\
\text { if } u \in V_{2}-V_{1} \text { and }(u, v) \in E_{2}-E_{1} \\
1-1+\left(\sigma_{1}(u) \vee \sigma_{2}(v)\right), \\
\text { if } u \in V_{1}, v \in V_{2} \text { and }(u, v) \notin E \text { (by 5.1) }
\end{array}\right. \\
= & \left\{\begin{array}{r}
\overline{\mu_{1}}(u, v) \text { if }(u, v) \in E_{1}-E_{2} \\
\overline{\mu_{2}}(u, v) \text { if }(u, v) \in E_{2}-E_{1} \\
\sigma_{1}(u) \vee \sigma_{2}(v) \text { if }(u, v) \notin E^{\prime}
\end{array}\right. \\
= & \left(\overline{\mu_{1}}+\overline{\mu_{2}}\right)(u, v) \quad
\end{aligned}
$$

\section{References}

[1] J.N. Morderson, P.S. Nair, Fuzzy Graphs and Fuzzy Hyper Graphs, Physics-Verlag, Heidelberg 2001.

[2] A. Nagoorgani, V.T. Chandrasekaran, A First Look at Fuzzy Graph Theory, Allied Pubications, India, 2010.

[3] A. Nagoorgani, J. Malarvizhi, On antipodal fuzzygraph, Applied Mathematical Sciences, 4, No. 43 (2010), 2145-2155.

[4] A. Rosenfeld, Fuzzygraphs, In: Zadeh, L.A. Fu, K.S.Shimura, M (Eds), Fuzzy sets and their Applications, Academic Press, Newyark (1975), 75-95.

[5] R. Seethalakshmi, R.B. Gnanajothi, Operations on antifuzzy graphs, Mathematical Sciences International Research Joournal, 5, No. 2 (2016), 210-214.

[6] R. Seethalakshmi, R.B. Gnanajothi, On antipodal antifuzzy graphs, International J. of Pure and Applied Mathematics, 112, No. 5 (2017), 47-55, doi: 10.12732/ijpam.v112i5.6.

[7] M.S. Sunithat, Sunil Mathew, Fuzzy graph theory: A survey, Annals of Pure and Applied Mathematics, 4, No. 1 (2013), 92-110.

[8] M.S. Sunitha, A. Vijayakumar, Complement of a fuzzy graph, Indian Journal of Pure and Applied Mathematics, 9, No. 33 (2002), 1451-1464.

[9] M.S. Sunitha, A. Vijayakumar, Some metric aspects of fuzzy graphs, In: Proceedings of the Conference on Graph Connections, Cochin University of Science and Technology, Cochin, India, January 28-31, 1998, 111-114. 